\section{RIKEN aids international structural genomics efforts}

SIR — We would like to respond to comments made in your News story ${ }^{\alpha}$ Big science' protein project under fire (Nature 443, 382; 2006) about Japan's Protein 3000 Project. This government project funds nine centres, including the RIKEN Structural Genomics/Proteomics Initiative, consisting of the RIKEN Genomic Sciences Center's Protein Research Group (http://protein.gsc. riken.jp) and the RIKEN SPring-8 Center.

First, we do not agree that the information gained is "of limited use" or, as one researcher is quoted as saying "a lot of it is junk". RIKEN has made major contributions to structures and structural models of functionally important proteins. It is expected to have determined 2,500 new structures by spring 2007 , or $5 \%$ of the entire Protein Data Bank (PDB). Nearly half of those determined so far were obtained using NMR, and consist of functional domains from biologically important (including disease-related, signal transduction and nucleic-acid-binding) human/mouse proteins. Multiple structures from each family were analysed to understand binding specificities. About 70\% of all NMR structures of human/mouse proteins deposited in the PDB in 2005 were from RIKEN.

The Protein 3000 project contributes significantly to the goals of the International Structural Genomics Organization (www. isga.org) by providing large numbers of templates that can be used to model other members of the protein families. On average, each NMR structure from RIKEN has contributed to about 300 new homology models, and each X-ray structure to about 200 new models at a level of $30 \%$ sequence identity. Quality assessment measures for RIKEN structures are similar to those for structures deposited in the PDB in 20002006 by traditional structural biology groups, according to Gaetano Montelione of the Northeast Structural Genomics Consortium (personal communication).

Second, we very strongly disagree with the comment that "A centre of that size should contribute to methodology, but there has been nothing." RIKEN has made seminal contributions to the development of methodologies and technologies. RIKEN has pioneered cell-free protein synthesis on a production scale, and developed technologies for extensive sample optimization process using the cell-free method. These technologies have been indispensable in solving the structures of many difficult proteins. More than 1,000 NMR structures have been determined from protein samples synthesized by RIKEN's implementation of the cell-free method. Additionally, at RIKEN, N. Kobayashi has developed the KUJIRA software for spectral analysis and P. Güntert has developed the CYANA software for automated protein structure analysis. RIKEN will be opening the NMR facility, together with these important technologies, to external scientists in 2007.

Shigeyuki Yokoyama ${ }^{\star}$, Thom as C. Terwilligert *RIKEN Genamic Sciences Center,

Yokohama 230-0045, Japan

†Los Alamos National Laboratory; on behalf of the ISGO Executive Committee This letter is also signed by:

SeikiKuramitsu, RIKENSPring-8 Center, Japan

Dino Moras, Institut de Génétiqueet de Blologie Maléculare et Cellubaire, France

Joel L Sussman, Isroel Structural Prateomics Center, Ispael

\section{Advances in biology reveal truth about prokaryotes}

SIR - Although we agree with William Martin and Eugene V. Koonin's point in Correspondence ("A positive definition of prokaryotes" Nature 442,$868 ; 2006$ ) about the validity of the term 'prokaryote', a term that Norman R. Pace has proposed abolishing ("Time for a change" Nature 441, 289; 2006), they have lost sight of the organismic biology forest for the molecular biology trees. The main differences between prokaryotic and eukaryotic cells probably relate to the original symbioses from which eukaryotes evolved.

Eukaryotes - whether protoctists, fungi, animals or plants - routinely open their membranes to take in (or let out) nuclear genomes, whole cells or other large particles, in processes such as ingestion, fertilization and hybridization. They reseal their membranes and live happily ever after. All eukaryotic sexuality requires cell fusion. Nearly all eukaryotic cell phenomena involve microscopically visible intracellular motility that never happens in prokaryotes.

We need to reassess our understanding of the course of evolution by recognition of the differences between unidirectional transfer of genetic material as the basis of prokaryotic sexuality - genophore DNA, viruses, plasmids - and parental cell fusion in eukaryotes. Roger Stanier and Cornelius van Niel's concept of 'prokaryote' was brilliantly recognized in 1927 by Boris Kozo-Polyansky, who only wrote in Russian. The word 'procariotique' was independently coined in 1925 by Edouard Chatton for cyanobacteria and all other bacteria including archaebacteria (J. Sapp International Microbiology 9,

$163-172 ; 2006)$. Because of the modern developments of biochemistry and molecular biology, electron microscopy and comparative genetics, the term 'prokaryote' is even more valid now than it was when first introduced. Michael F. Dolan, Lynn Margulis Department of Geosciences, University of Massachusetts-Amherst, 611 North Pleasant St, Amherst, Massachusetts 01003-9297, USA

\section{Pollution analysis flawed by statistical model}

SIR - I found your Special Report on airpollution control in the United States ( ${ }^{\alpha}$ The politics of breathing " Nature 444, 248-249; 2006) to be generally well balanced. I would like to point out, however, that there is by no means universal agreement among scientists that air pollution at contemporary USlevels affects human health. I am one of the sceptics.

The report seems to take at face value the conclusion of "two large, well-respected epidemiological studies", that every additional microgram of fine particles per cubic metre in the air causes tens of thousands of deaths a year in the United States. Yet joint pollutant analyses - with sulphur dioxide and either sulphates or fine particles both induded in the statistical models - show that sulphur dioxide is associated with mortality; fine particles are not (D. Krewski et al. Reanalysis of the Harvard Six Cities Study and the American Cancer Society Study of Particulate Air Pollution and Mortality Health Effects Institute, 2002). The association of sulphur dioxide with mortality remains unexplained, as there is no plausible biological mechanism by which it could be causing death.

Further, the pollution studies mentioned used the proportional hazards model for analyses of the data. This model assumes that the relative risks of air pollution and potential confounders remain constant over time. It is clear, however, that the basic assumption of proportionality of hazards is satisfied neither for air pollution nor for a strong potential confounder, cigarette smoking (S.H.

Moolgavkar Inhal. Toxicol. 18, 93-94;2006). Use of this model when the assumption of proportionality of hazards is violated can have serious consequences for the inferences drawn from the data. It may, for example, explain the very different results of observational epidemiological studies of hormone replacement therapy in the $1990 \mathrm{~s}$ and the recently concluded Women's Health Initiative randomized trial (R. L. Prentice et al. Am. J. Epidemiol. 162, 404-414; 2005). Departing from assumptions of proportionality of hazards for potential confounders may also bias the estimates of main effects in cohort studies, particularly when the confounder is a strong risk factor. In air-pollution studies, the use of a manifestly wrong model to adjust for confounding by smoking probably biases the estimates of small air-pollution effects on mortality, although the direction of the bias will depend upon the structure of the correlation between smoking and air pollution.

We do not currently have the methods to reliably estimate small environmental risks. Suresh Moolgavkar Fred Hutchinson Cancer Research Center, 1100 Fairview Avenue N, Seattle, Washington 98109 , USA 